\title{
Adventitial Cystic Disease of the Femoral Vein: a Case Report with the CT Venography
}

Jae Young Seo, MD'

Dong Jin Chung, MD²

Ji Hyung Kim, MD

\author{
Index terms: \\ Veins, iliac \\ Veins, stenosis or obstruction \\ Computed tomography (CT), \\ angiography
}

DOI:10.3348/kj.2009.10.1.89

Korean J Radiol 2009; 10:89-92 Received February 19, 2008; accepted after revision October 9, 2008.

'Department of Radiology, University of Konyang College of Medicine, Daejeon 302-718, Korea; '2Department of Radiology, Gachon University Gil Hospital, Incheon 405-760, Korea; ${ }^{3}$ Department of Radiology, Sam Hospital, Anyang 430-015, Korea

Address reprint requests to: Dong Jin Chung, MD, Department of Radiology, Gachon University Gil Hospital 1198 Guwol-dong, Namdong-gu, Incheon 405-760, Korea.

Tel. (8232) 460-3057

Fax. (8232) 460-3065

e-mail: bookdoo7@chollian.net
Fewer than 20 cases of adventitial cystic disease of the vein have been reported in the worldwide literature. This small number of reported cases may be due not only to the disease's low incidence, but also to the difficulty in making the proper diagnosis. Many techniques have been used to investigate this disease, but venography has been the traditional diagnostic tool. In this report we present a case of adventitial cystic disease that was well demonstrated by CT venography.
A dventitial cystic disease of the vein is a rare condition. The arterial variety of adventitial cystic disease has often been described in the popliteal artery and this is characterized by a mucinous cyst located in the adventitia of the artery, with the contents of the cyst resembling the contents of a ganglion. In this report, we discuss the case of a 69 -year-old man who presented with a swollen leg secondary to obstruction of the common femoral vein. We performed CT venography and ultrasound and this led to excision of a cyst and vein repair via a vessel graft. As a result, the patient made a full recovery. We also discuss the pathology and the diagnostic methods for this condition.

\section{CASE REPORT}

A 69-year-old man presented with a one month history of swelling of the left lower extremity. He had no specific past medical history and no history of trauma. No other abnormality was found on physical examination and we suspected that he suffered with deep vein thrombosis. Ultrasonography showed a cystic mass containing hypoechoic materials attached to the left common femoral vein (Fig. 1A). For further evaluation, we performed CT venography using 64 channel multi-detector computed tomography (Aquilion, Toshiba, Japan) with the patient in the supine position. A 22gauge intravenous cannula was placed in the dorsal vein of the left foot, and a tourniquet was placed around the left knee. Forty milliliters of a contrast medium diluted with 60 milliliters of saline was automatically injected via a Y adapter into the left leg at a rate of $3 \mathrm{cc} / \mathrm{sec}$. The optimal scan delay for the venous phase was determined by use of bolus tracking at the left popliteal vein. The axial and coronal reconstruction images showed a $1.7 \mathrm{~cm}$ sized cystic mass compressing the left common femoral vein along with dilated medial circumflex femoral and obturator veins that provided collateral circulation for the occluded left common femoral vein (Fig. 1B, C). A 3D volume rendering image showed an abrupt tapered disconnection of the left common femoral vein along with the formation of multiple collateral vessels (Fig. 1D). The patient underwent an operation because of his swollen leg. Surgical exploration showed an 
approximately $1.5 \mathrm{~cm}$ sized cystic mass over the anterior surface of the left common femoral vein. A longitudinal venostomy was performed at this location and a gelatinous material exuded from the mass and this gelatinous material could also be aspirated from the mass. A 10-cm long segment of the left common femoral vein/distal iliac vein was resected and a Gore-Tex graft was interposed.

Microscopically, a cystic widened adventitial layer of the vein showed adventitial hemorrhage, fibrin deposition and focal aggregation of foamy histiocytes and focal myxoid degeneration (Fig. 1E, F). CT venography was performed again after the operation for a follow up evaluation. The cystic mass in the left inguinal areas was gone, as were the multiple collateral vessels (Fig. 1G). The final diagnosis was adventitial cystic disease.

\section{DISCUSSION}

Adventitial cystic disease is an unusual condition of an

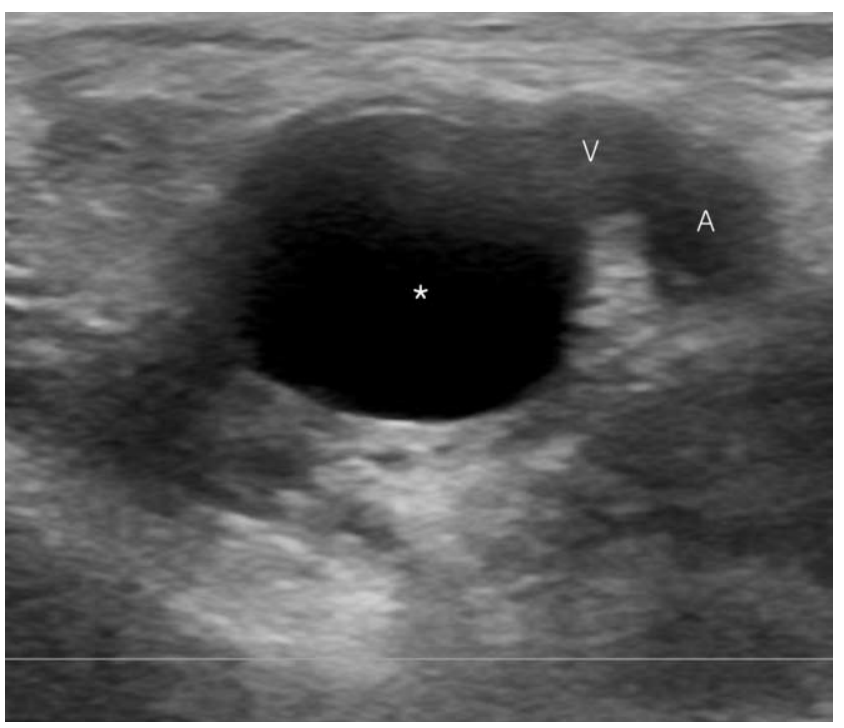

Fig. 1. US of adventitial cystic disease in 69-year-old man.

A. US showed relationship between cystic mass and left common femoral vein. Cystic mass ( $\left.{ }^{*}\right)$ that contained hypoechoic materials is attached to medial side of left common femoral vein (v).

B. Venous phase axial CT scan shows approximately $1.7 \mathrm{~cm}$ sized round, nonenhancing cystic mass (long arrow) compressing left common femoral vein with dilated medial circumflex femoral and obturator veins, which provide collateral circulation.

C. Coronal reconstruction image shows low attenuation nonenhancing mass (white arrow) in left common femoral vein.

D. Posterior view of three dimensional volume rendering image of left common femoral vein showing near complete obstruction of left common femoral vein (white arrow) with formation of multiple collateral vessels.
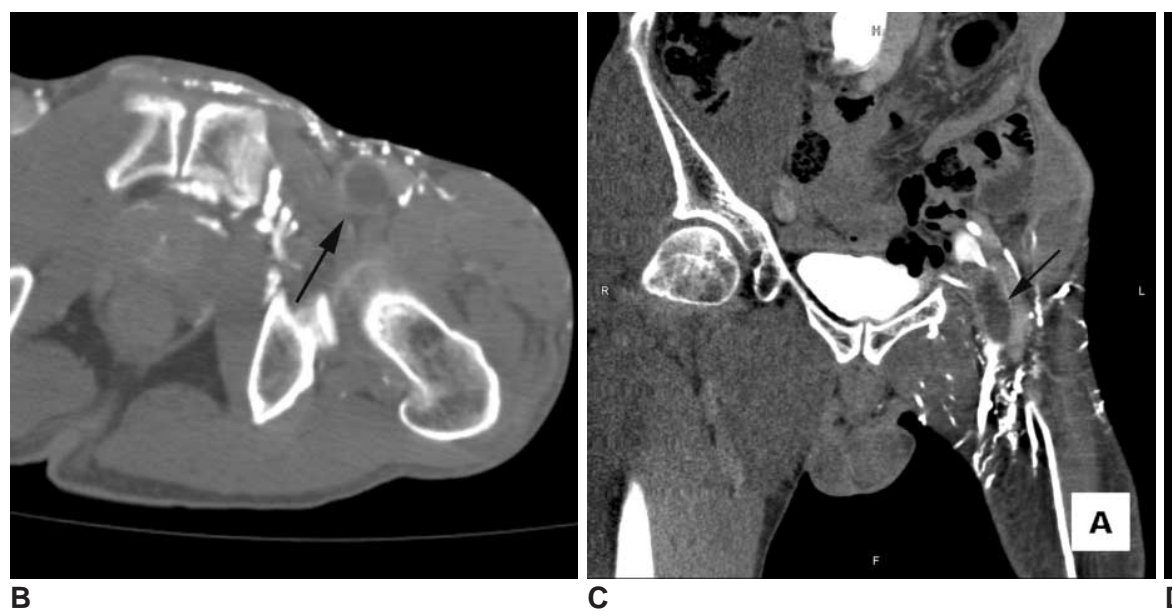


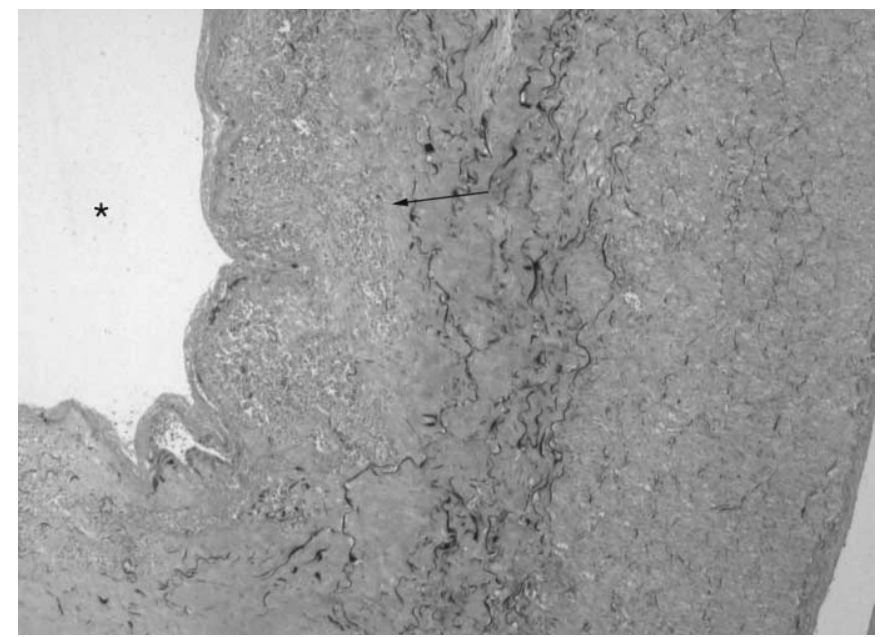

E

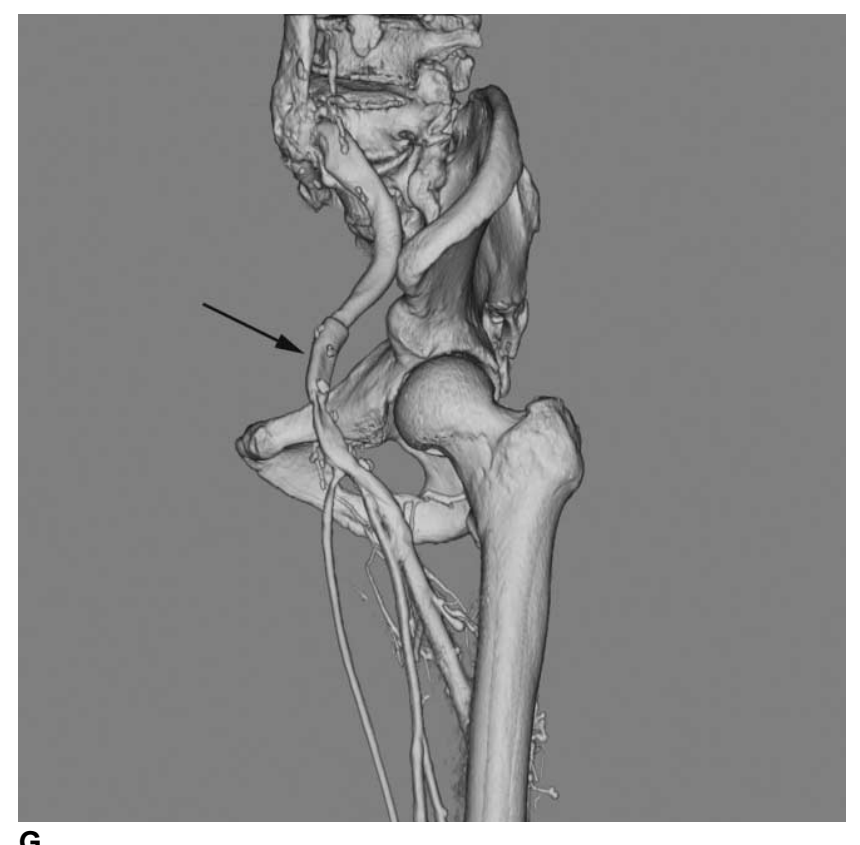

G

adventitial cystic disease of the arteries is more frequent in men, it is predominantly located in the popliteal artery and it clinically presents with intermittent claudication. In contrast to arterial adventitial cystic disease, the venous variety rarely affects the popliteal segment. The venous variety occurs with an equal frequency in both sexes and it most often involves the common femoral vein and causes swelling of the affected limb.

The etiology of adventitial cystic disease remains unclear, but there are several theories $(3,5,7-9)$. Repeated microtrauma, ectopic aganglionosis, degeneration of the adventitia due to connective tissue diseases and developmental theory have all been discussed in the medical literature. Histopathologically, the cyst may be uniloculated or multiloculated $(2,4)$. The disease process produces an expanding cyst that destroys the elastic tissue between the

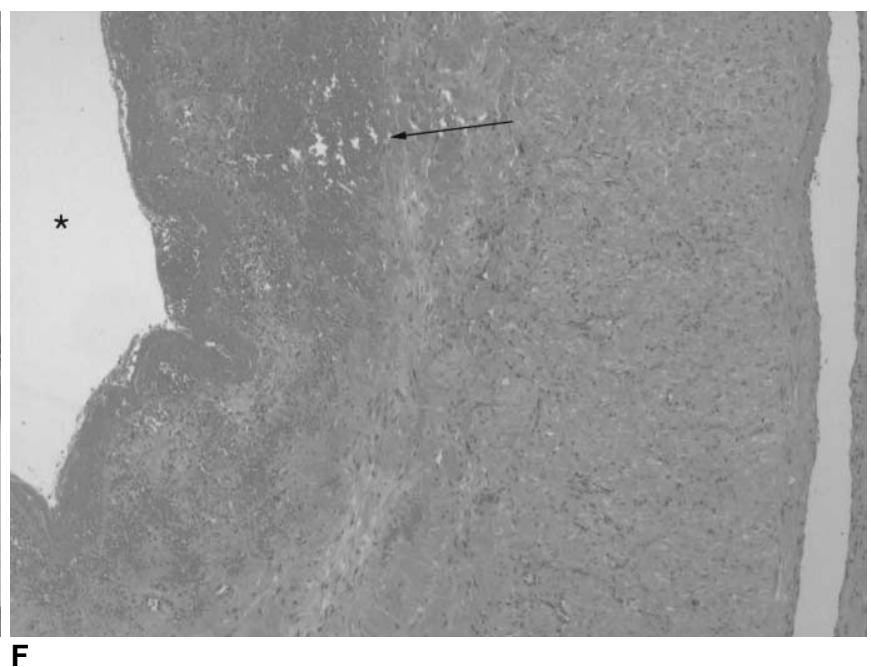

Fig. 1. US of adventitial cystic disease in 69-year-old man.

E. Photomicrograph of excised left common femoral vein. X40; Elastin Van Gieson stain reveals widening of adventitial layer of vein and replacement of elastic tissue by fibrous connective tissue (arrow), which is all consistent with cystic adventitial disease. Cyst was removed (asterisk).

F. Photomicrograph of excised left common femoral vein. $\times 40$; Hematoxylin \& Eosin stain shows adventitial hemorrhage and fibrin deposition within widened adventitial layer of vein (arrow). * = cyst G. Postoperative CT venography of patient's adventitial cystic disease. Lateral view of three dimensional volume rendering image showing that left common femoral vein (arrow) runs through previous cystic mass site and multiple collateral vessels have disappeared.

medium and the adventitia of the vessel wall, and the elastic tissue is replaced with fibrous connective tissue. There is usually no acute or chronic inflammation. The cyst is lined by fibrous connective tissue and the cyst contains an eosinophilic mucoid gel that consists of mucoproteins and mucopolysaccharides.

The diagnosis of adventitial cystic disease of the vein can be suspected on the basis of the patient history, the results of a physical examination and the image findings (6). Venography shows a smooth-walled stenosis that may be curvilinear with an hourglass or spiral configuration (1, 3, $4,10)$. The important CT features in this case included eccentric compression of the lumen owing to a thin-walled cystic mass with an enhanced rim. Predictably, the mucinous cyst contents showed no enhancement and the contents had intermediate attenuation values between that 
of water and muscle. Ultrasonography may show a typical hypoechoic fluid filled cyst with a posterior acoustic window and this may allow ultrasound-guided treatment (5). Duplex US may show stenosis and blood flow changes in the prestenotic and poststenotic regions. Yet ultrasonography cannot display the distribution of the collateral vessels seen on $\mathrm{CT}$ and $\mathrm{CT}$ venography. Because of the collateral vessels, it is much more difficult to determine the exact anatomic relationships around the mass. Performing $\mathrm{CT}$ venography in patients with venous adventitial cystic disease can reveal the site and the extent of the obstruction, and it may show a classic scalloped appearance or hourglass narrowing caused by the extrinsic compression of the vessel lumen. CT venography is superior to traditional venography for making the diagnosis because the cystic mass can be directly observed regardless of the degree of obstruction. The involved vessel and also the structures such as collateral vessels can also be visualized during this procedure, and follow up evaluations are routine and not so difficult to perform.

In summary, adventitial cystic disease of the vein is a rare malady, but it should be suspected for patients with the symptoms of deep vein thrombosis, and especially when the diagnostic investigations indicate an extrinsic mass. CT venography is useful for demonstrating the lesion and increasing the specificity of the radiologic diagnosis, and this modality can be routinely used for the postoperative follow-up evaluation.

\section{References}

1. Wilbur AC, Woelfel GF, Meyer JP, Flanigan DP, Spigos DG. Adventitial cystic disease of the popliteal artery. Radiology 1985;155:63-64

2. Atkins HJB, Key JA. A case of myxomatous tumour arising in the adventitia of the left external iliac artery. Br J Surg 1947; 34:426-427

3. Velasquez G, Zollikofer C, Nath HP, Barreto A, CastanedaZuniga W, Formanek A, et al. Cystic arterial adventitial degeneration. Radiology 1980;134:19-21

4. Do DD, Braunschweig M, Baumgartner I, Furrer M, Mahler F. Adventitial cystic disease of the popliteal artery: percutaneous US-guided aspiration. Radiology 1997;203:743-746

5. Dix FP, McDonald M, Obomighie J, Chalmers N, Thompson D, Benbow EW, et al. Cystic adventitial disease of the femoral vein presenting as deep vein thrombosis: a case report and review of the literature. J Vasc Surg 2006;44:871-874

6. Maldonado-Fernandez N, Lopez-Espada C, Moreno-Escobar J, Martinez-Gamez J, Rodriguez-Morata A, Garcia-Rospide V. Recurring adventitial cyst in the left external iliac vein. EJVES Extra 2004;8:10-14

7. Cho K, Shin TB. A case of adventitial cystic disease of the femoral vein. J Korean Soc Vasc Surg 2005;21:186-189 [Korean]

8. Gagnon J, Doyle DL. Adventitial cystic disease of common femoral artery. Ann Vasc Surg 2007;21:84-86

9. Fukui S, Paraskevas N, Lafaurie C, Soury P, Gigou F, Petit MD, et al. Cystic formation compressing the femoral vein: synovial hip joint or adventitial cyst. EJVES Extra 2004;8:1-4

10. Jasinski RW, Masselink BA, Partridge RW, Deckinga BG, Bradford PF. Adventital cystic disease of the popliteal artery. Radiology 1987;163:153-155 